\title{
Detection of Chronic Kidney Disease in High Risk Groups Attending In a Tertiary Care Hospital of North East India
}

\author{
Dr. Rajesh Kishore Debbarma ${ }^{1}$, Dr. Debasis Ray ${ }^{2}$ \\ ${ }^{I}$ MD, Associate professor Department of Medicine, Agartala Government Medical College, kunjaban, \\ Agartala, Tripura 799006 \\ ${ }^{2}$ MD, Associate professor Department of Pharmacology, Agartala Government Medical College, kunjaban, \\ Agartala, Tripura 799006
}

\begin{abstract}
Chronic Kidney Disease (CKD) is becoming a major global health problem. In India, diabetes and hypertension are responsible for $40 \%$ to $50 \%$ of all cases of CKD. The present study was carried out with the objective to detect the prevalence of asymptomatic CKD in high risk groups like diabetes and hypertension. Total 86 patients of age group above 18 of both sexes were selected randomly. Glomerular Filtration Rate (GFR) was estimated by Modification of Diet in Renal Disease (MDRD) 6 equation. Observation revealed that 15 cases had GFR >90, 40 cases had GFR 60-90. And 31 cases had GFR $<60 \mathrm{ml} / \mathrm{min}$ per $1.73 \mathrm{~m}^{2}$. Main age groups affected were $>50$ years $(80 \%)$.CKD was seen in $40 \%$ of hypertensive and $27.8 \%$ of diabetic subjects. Result of this study is comparable with the study of Timothy HM et al who has also shown that $20.4 \%$ of his subjects had CKD, and higher prevalence among hypertensive than diabetic (69\% and 30\% respectively). The mean BMI of subjects having CKD is $23.8 \pm 3.159$ and mean BMI of subjects not having CKD is 25.1 \pm 4.353 . This observation shows that patients with CKD are having high normal BMI.CKD is a recognized risk factor of ESRD and cardiovascular death, which is a leading cause of death in developing countries. An increase in BMI is a significant factor independently associated with CKD. In the present study, the differences in BMI are relatively small and have an insignificant $P$ value between those with and without $C K D$. An interventional approach is necessary to prevent development of CKD at every level, especially in high risk groups to prevent CKD and development of End Stage Renal Disease (ESRD).
\end{abstract}

\section{Introduction:}

Chronic Kidney Disease (CKD) is becoming a major global health problem. In India there is a rising burden of chronic diseases like hypertension and diabetes. The increase number of CKD patient can be partially attributed to the epidemic of hypertension and diabetes and aging population. India has the largest number of diabetes in the world. The prevalence of hypertension has been reported to range between $20-40 \%$ in urban adult and $12-17 \%$ among the rural adults. It is estimated that $25-40 \%$ of these patients are likely to develop CKD, with significant percentage requiring Renal Replacement Therapy (RRT)

Prevalence of CKD is higher in patients with cardiovascular disease. Early CKD constitutes a significant risk factor for cardiovascular events and death. Both the frequency of cardiovascular complication and the progression of CKD can be ameliorated in these patients by appropriate intervention. For all these reason, healthcare providers should evaluate their patients for the presence of CKD as part of preventive care and treatment strategies ${ }^{3}$.

Early diagnosis of CKD is possible and prevention of kidney failure requiring RRT is feasible. Information on incidence and outcome of CKD in India is not available. It is estimated that lakh patients develop End Stage Renal Disease (ESRD) every year in India, ${ }^{2,4}$ only $3 \%$ to $5 \%$ of all patients with ESRD in India get some form of $\mathrm{RRT}^{5}$. Thus it is important to diagnose kidney disease early and planning for prevention of CKD is the only practical solution in India ${ }^{2,4,5}$.

It appears that even in India, diabetes and hypertension are responsible for $40 \%$ to $50 \%$ of all cases of chronic renal failure. Screening for these two diseases and CKD is simple and easy to perform. The best approach is to start screening for CKD in a high - risk group, like first - degree relatives of patients with diabetes, hypertension and CKD and simultaneously making a platform to run the program through the existing health care system of the country ${ }^{5}$.

CKD is defined as structural or functional abnormalities of kidney that persists for at least 3 months and are manifested by either kidney damage (most frequently detected as persistent albuminuria; $>30 \mathrm{mg}$ albumin / gm creatinine) or a decreased GFR $\left(<60 \mathrm{ml} / \mathrm{min}\right.$ per $\left.1.73 \mathrm{~m}^{2}\right)$. Because techniques commonly used to estimate renal function often fail to detect patients with mild to moderate reduction in GFR and because screening for albuminuria is not constantly performed, CKD in many patients remains unidentified ${ }^{3}$.

The Kidney disease Outcome Quality Initiative (K / DOQI) guidelines recommended screening of CKD patients who have diabetes mellitus, hypertension, recurrent UTI etc. Serum creatinine is a highly flawed measure of 
glomerular filtration rate (GFR). The Modification of Diet in Renal Disease (MDRD) formula is a better estimate of GFR than those derived from 24 hour urinary creatinine clearance. Formula based estimate of GFR are now referred as estimated GFR (eGFR) ${ }^{6}$.

Screening patients at risk for CKD relies on the detection of functional abnormalities using readily available, inexpensive laboratory tests. Current guidelines recommended screening for kidney disease with a serum creatinine measurement for use of GFR estimation and analysis of random urine sample for albuminuria. Significant kidney disease can present with decreased GFR or protenuria, or both ${ }^{7}$.

CKD typically have a variable latent period during which the disease is present and asymptomatic. Although it is not clear when CKD becomes symptomatic, Substantial loss of renal function can occur before clinical events associated with CKD become apparent. Anemia, which may be symptomatic, is generally observed among CKD patients when the GFR falls below $60 \mathrm{ml} / \mathrm{min}$. With GFR less than $30 \mathrm{ml} / \mathrm{min}$, symptoms associated with uremia become frequent. There is substantial range of GFR during which patients with CKD, defined by a GFR of $90 \mathrm{ml} / \mathrm{min}$ or less, may be asymptomatic and where screening is justified ${ }^{8}$.

Both albuminuria and decrease in eGFR were used as 2 basic markers for the new classification of CKD proposed by the Kidney Disease Outcome Quality Initiative of the National Kidney foundation?.

CKD is a worldwide under diagnosed public health problem with increasing incidence and prevalence that has high costs and poor outcome. Unfortunately, CKD in its earliest stages is usually an asymptomatic condition which progresses to its end stage over a period of several years and is diagnosed late. Continuous increase in the number of patients with end stage renal disease demands early detection of CKD. Therefore, strategies to reduce the incidence of End Stage Renal Disease (ESRD) require effective methods of screening early in the disease process 9 .

\section{Material \& Methods:}

The present cross sectional study was carried out with the objective to detect the prevalence of person suffering from asymptomatic CKD in high risk group that would help to allocate detected asymptomatic cases to receive appropriate therapy, so that it can halt the progression of CKD. Patients who were suffering from diabetes and hypertension were selected for this study. Total 86 patients of age group above 18 of both sexes were selected randomly from outpatient pool of Department of Medicine of a tertiary care hospital of a North Eastern State.

The known CKD patients, patients with history of acute renal failure, pregnant ladies and seriously ill patients with hypertension and / or diabetes mellitus were excluded from the present study. Diabetes mellitus was diagnosed by the criteria laid down by American Diabetic Association which are follows: symptoms of diabetes plus random blood glucose $>200 \mathrm{mg} / \mathrm{dl}$ or fasting plasma blood glucose $\geq 126 \mathrm{mg} / \mathrm{dl}$ or two hour plasma glucose $\geq 200 \mathrm{mg} / \mathrm{dl}$ during oral glucose tolerance test. Serum albumin was estimated by BCG dye method, end point with fully automated biochemical anlyzer ${ }^{10}$ serum creatinine was estimated by Jaffes method, initial rate with fully automated biochemical analyzer ${ }^{11}$. The GFR was estimated using a simplified prediction equation derived from the Modification of Diet in Renal Disease (MDRD) Study ${ }^{12}$ and given by the following equation.

$$
\begin{aligned}
\mathrm{GFR}=186.3 & \times\left(\text { serum }_{\text {creatinine }}-1.154\right. \\
& \times\left(\mathrm{age}^{-0.203}\right) \\
& \times 1.212(\text { if African American }) \\
& \times 0.742(\text { if female })
\end{aligned}
$$

Data were analyzed by using statistical package for social sciences (SPSS)

\section{Observations}

In our study a total of 86 samples were included. Out of 86 enrolled subjects 36 cases were diabetes mellitus $(41.9 \%)$ and 50 cases were hypertensive $(58.1 \%)$.

Out of 86 patients 33 cases were female $(38.4 \%)$ and 53 cases were male $(61.6 \%)$. out of 33 female cases 10 cases were diabetic $(30.3 \%)$ and 23 cases were hypertensive $(69.7 \%)$. Whereas out of 53, 26 male cases were diabetic $(49.1 \%)$ and 27 cases were hypertensive $(50.9 \%)$, Tabulated in table 1.

\begin{tabular}{|l|cc|c|}
\hline Sex of patients & \multicolumn{2}{|c|}{ Diagnosis } & Total \\
& Diabetes & Hypertension & \\
\hline Female & 10 & 23 & 33 \\
$\%$ within sex of patients & $30.3 \%$ & $69.7 \%$ & $100 \%$ \\
\hline Male & 26 & 27 & 53 \\
\% within sex of patients & & & $100 \%$ \\
\hline Total & $49.1 \%$ & $50.9 \%$ & 86 \\
\hline
\end{tabular}

Table1: sex and diagnosis of patients cross tabulation. 
Estimated GFR in our subjects revealed that 15 cases had GFR >90, 40 cases had GFR 60-90. And 31 cases had GFR $<60 \mathrm{ml} / \mathrm{min}$ per $1.73 \mathrm{~m}^{2}$ (Table 2 ).

\begin{tabular}{|l|l|l|}
\hline Stages & GFR & eGFR \\
\hline 1 & $>90$ & 15 \\
\hline 2 & $60-90$ & 40 \\
\hline 3 & $30-59$ & 30 \\
\hline 4 & $15-29$ & 01 \\
\hline 5 & $<15$ & 00 \\
\hline
\end{tabular}

Table 2 showing estimated GFR of patients

$\mathrm{CKD} \geq 3$ was seen more in the age group of $>50$ years $(n=25)$ and only 6 cases in the age group of $<50$ years. (table 3 )

\begin{tabular}{|l|l|}
\hline Age group & CKD $\geq 3$ \\
\hline$<50$ years & 6 \\
\hline$>50$ years & 25 \\
\hline
\end{tabular}

Table 3-distribution of $\mathrm{CKD} \geq 3$ in different age group

CKD were seen in $40 \%$ of hypertensive and $27.8 \%$ of diabetic subjects. Comparison of presence of CKD in hypertensive and diabetic subjects shows that $42 \%$ of hypertensive subjects has GFR $<60 \mathrm{ml} / \mathrm{min}$ per $1.73 \mathrm{~m}^{2}$ and $27.8 \%$ of diabetic patients has GFR $<60 \mathrm{ml} / \mathrm{min}$ per $1.73 \mathrm{~m}^{2}$ tabulated in table 4

\begin{tabular}{|c|c|c|c|c|c|c|}
\hline Diagnosis & & $>90$ & $60-90$ & $30-59$ & $15-30$ & $<15$ \\
\hline Diabetes & $\begin{array}{l}\text { Count } \\
\% \text { within } \\
\text { diagnosis }\end{array}$ & $\begin{array}{l}9 \\
25 \%\end{array}$ & $\begin{array}{l}17 \\
47.2 \%\end{array}$ & $\begin{array}{l}10 \\
27.8 \%\end{array}$ & $\begin{array}{l}0 \\
0 \%\end{array}$ & $\begin{array}{l}0 \\
0 \%\end{array}$ \\
\hline Hypertension & $\begin{array}{l}\text { Count } \% \text { within } \\
\text { diagnosis }\end{array}$ & $\begin{array}{l}6 \\
12 \% \\
\end{array}$ & $\begin{array}{l}23 \\
46 \% \\
\end{array}$ & $\begin{array}{l}20 \\
40 \% \\
\end{array}$ & $\begin{array}{l}1 \\
2 \% \\
\end{array}$ & $\begin{array}{l}0 \\
0 \% \\
\end{array}$ \\
\hline Total & & 15 & 40 & 30 & 1 & 0 \\
\hline
\end{tabular}

.Table 4: diagnosis of patients, eGFRcross-tabulation.

The mean BMI of subjects having CKD is $23.7740000, \mathrm{SD}=3.159$ and mean BMI of subjects not having CKD is $25.100, \mathrm{SD}=4.353$. The two-tailed $\mathrm{P}$ value equals 0.1442 and considered to be not statistically significant. With a $95 \%$ confidence interval.

\begin{tabular}{|r|r|r|}
\hline Group & GFR $<60 \mathrm{ml} / \mathrm{min}$ per $1.73 \mathrm{~m}^{2}$ & GFR $>60 \mathrm{ml} / \mathrm{min}$ per $1.73 \mathrm{~m}^{2}$ \\
\hline Mean BMI & 23.7740000 & 25.1000000 \\
\hline SD & 3.1592200 & 4.3532600 \\
\hline
\end{tabular}

\section{Discussion}

CKD affects approximately 19 million adult Americans. The burden of CKD in India cannot be assessed accurately. The approximate prevalence of CKD is 800 per million populations. ${ }^{13}$ Evidence suggests that progression to kidney failure can be delayed or prevented by controlling blood sugar levels and blood pressure and by treating proteinuria. Unfortunately, CKD often is overlooked in its earliest, most treatable stages. Primary care physicians have an important role in detecting CKD early, in instituting measures to slow disease progression, and in providing timely referral to a nephrologist. ${ }^{14}$

Burden of hypertension and diabetes is increasing In India. The prevalence of hypertension has been reported to range between $20-40 \%$ in urban adult and $12-17 \%$ among the rural adults. It is estimated that $25-$ $40 \%$ of these patients are likely to develop $\mathrm{CKD}^{1}$. Gabriel CD et al in a cross sectional analysis of more than 2500 diabetic patients has demonstrated that the prevalence of CKD was $22.9 \% .^{15}$

In our present study we have observed that the prevalence of asymptomatic CKD in high risk group was $31 \%$ and out of these $67 \%$ are hypertensive and $33 \%$ are diabetic. So, the prevalence of CKD is higher in hypertensive than diabetic subjects. Result of this study is comparable with the study of Timothy HM et al who has also shown that in his 402 high risk subjects with hypertension, diabetes, 20.4\% had CKD, and higher prevalence among hypertensive than diabetic (69\% and $30 \%$ respectively) ${ }^{16}$

Deidra $\mathrm{C}$ et $\mathrm{al}{ }^{17}$ have determined the prevalence of CKD within blood pressure categories in 17,794 adults. Prevalence of CKD among those with pre- and undiagnosed hypertension was $17.3 \%$ and $22.0 \%$, respectively, compared to $27.5 \%$ with diagnosed hypertension 
Hida $\mathrm{M}$ et al $^{18}$ has studied Age distribution at dialysis induction among patients with CKD in 579 cases. Age distribution differed depending on the primary renal diseases and sex. However, the 50-59 year-old group had the most cases ${ }^{18}$. We have also found that prevalence of CKD was more in the age group of $>50$ years $(\mathrm{n}=25)$ than $<50$ years $(\mathrm{n}=6)$

Gelber RP et al ${ }^{19}$ after an average 14-year follow-up of 1,377 healthy participants demonstrated that CKD was present in $12.4 \%$ participants. Higher baseline BMI $(>26.6 \mathrm{~kg} / \mathrm{m} 2)$ was associated consistently with increased risk for CKD. Compared with participants in the lowest BMI quintile $(<22.7 \mathrm{~kg} / \mathrm{m} 2)$ in our present study we have found that The mean BMI of subjects having CKD is $23.8 \pm 3.159$ and mean BMI of subjects not having CKD is $25.1 \pm 4.353$. This observation shows that patients with CKD are having high normal BMI.

CKD has recently been recognized not only as a risk factor of ESRD, but also of cardiovascular death which is a leading cause of death in developing countries. Diabetes and Hypertension has been clearly shown to be an important risk factor for future reduction in GFR and development of ESRD. An increase in BMI is a significant factor independently associated with CKD. In the present study, the differences in BMI or the percentages of obese subjects are relatively small between those with and without CKD. An interventional approach is necessary to prevent development of CKD at every level, especially in high risk groups to prevent CKD and development of ESRD.

Lastly, we should mention the limitations of the present study. First, the number of subjects enrolled was small and secondly study has only included the patients with diabetes mellitus and hypertension, missing other risk groups. Next, this is simply an observational study examining the cross-sectional relationship between hypertension, diabetes mellitus and CKD. An interventional, follow-up study involving a large cohort with various risk factors is required to have a better analysis of the problem to prevent CKD and its outcome in our state.

\section{Reference:}

[1]. $\quad$ Narula B AS. Chronic Disease: The Looming Threat. MJAFI 2008; 64: 2-3.

[2]. Modi GK and Jha V. The incidence of end stage renal disease in India: A population - based study. Kidney International 2006; 70: 2131-2133.

[3]. Frank CB, Thomas HH, Elie K, Mark MM Sharon MM. Detection of Chronic Kidney Disease in patients with or at increased risk of cardiovascular Disease. Hypertension 2006; 48: 751-755.

[4]. Moorthy AV. Early detection of Kidney disease is Important. India's National Magazine 2004; 21: 5-18.

[5]. Agarwal SK. Chronic Kidney Disease and its Prevention in India. Kidney International 2005; 68: 41-45.

[6]. MacGregor MS, Boag DE, Innes A. Chronic Kidney Diseases: Evolving strategies for detection and management of impaired renal function. QJM 2006; 99: 1-19.

[7]. Gansevoot RT, Bakker SJL and Jong PE. Early Detection of Progressive Chronic Kidney Disease: Is It Feasible? J Am Soc Nephrology 2006; 17: 1218-1220.

[8]. William M, McCleln S, Ramirez PB, Claudine J. Screening for Chronic Kidney Disease, Unresolved Issues. J Am Soc. Nephrool 2003; $14: 81-87$.

[9]. Krol E, Rutkowski B, Czekalski S, Sulowicz W and Wiecek A. Early Detection of Chronic Kidney Disease: Results of the Pol Nef Study. Am J Nephrol 2009; 29: 264-273.

[10]. Doumas BT,Arendas RL,Pinto PC.Standard method of clinical chemistry:7:175-189:1978

[11]. Bowers LD. Clinical Chemistry;26: 551:1980

[12]. A.S. Levey, J.P. Bosch, J.B. Lewis, T. Greene, N. Rogers and D. Roth. A more accurate method to estimate glomerular filtration rate from serum creatinine: a new prediction equation. Modification of Diet in renal disease study group: Ann Intern Med 1999 Mar 16; 130 (6): 471-70.

[13]. S.K. Agarwal , R.K. Srivastava. Chronic Kidney Disease in India: Challenges and Solutions. Nephron Clin Pract 2009; 111; 97203.

[14]. Susan S, Bernadette P. Detection and Evaluation of Chronic Kidney Disease. American Family Physician 2005; 72(9):1723-1732.

[15]. Gabriel Coll-de-Tuero1, Manel Mata. Chronic kidney disease in the type 2 diabetic patients: prevalence and associated variables in a random sample of 2642 patients of a Mediterranean area. BMC Nephrology 2012; 1471-2369-13:87

[16]. Timothy HM, Olivia C. Screening for chronic kidney disease in Australia:a pilot study in community and workplace. Kidney international: 2010; 77:9-16

[17]. Deidra C. Crews, Laura C. P. Prevalence of Chronic Kidney Disease in Persons with Undiagnosed or Prehypertension in the United States. Hypertension: May 2010; 55(5): 1102-1109.

[18]. Hida M, Saito H, Wakabayashi T, Satoh T. Age and sex distribution in chronic renal failure patients at dialysis induction. Tokai J Exp Clin Med. 1985 Dec; 10(6):581-8.

[19]. Gelber RP, Kurth T. Association between body mass index and CKD in apparently healthy men. Am J Kidney Dis. 2005; 46(5):871-80 